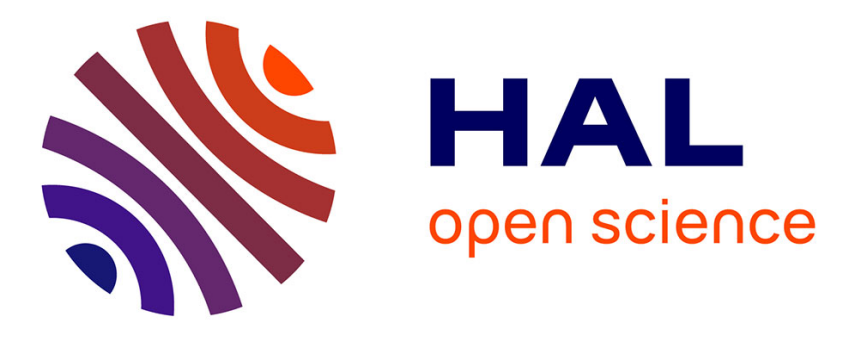

\title{
Self-consistent vertical transport calculations in AlxGa1-xN/GaN based resonant tunneling diode
}

\author{
A. Rached, A. Bhouri, S. Sakr, J.-L. Lazzari, H. Belmabrouk
}

\section{To cite this version:}

A. Rached, A. Bhouri, S. Sakr, J.-L. Lazzari, H. Belmabrouk. Self-consistent vertical transport calculations in AlxGa1-xN/GaN based resonant tunneling diode. Superlattices and Microstructures, 2016, 91, pp.37-50. 10.1016/j.spmi.2015.12.035 . hal-03150265

\section{HAL Id: hal-03150265 \\ https://hal.science/hal-03150265}

Submitted on 23 Feb 2021

HAL is a multi-disciplinary open access archive for the deposit and dissemination of scientific research documents, whether they are published or not. The documents may come from teaching and research institutions in France or abroad, or from public or private research centers.
L'archive ouverte pluridisciplinaire HAL, est destinée au dépôt et à la diffusion de documents scientifiques de niveau recherche, publiés ou non, émanant des établissements d'enseignement et de recherche français ou étrangers, des laboratoires publics ou privés. 


\title{
Self-consistent vertical transport calculations in $A l_{x} G a_{1-x} N / G a N$ based resonant tunneling diode
}

\author{
A. Rached ${ }^{1}$, A. Bhouri' ${ }^{1}$, S. Sakr², J.-L. Lazzari ${ }^{3}$, and H. Belmabrouk ${ }^{1}$ \\ ${ }^{1}$ Laboratoire d'Electronique et Microélectronique, Faculté des Sciences de Monastir, \\ Université de Monastir, 5019 Monastir, Tunisia. \\ ${ }^{2}$ Lebanese International University, Mouseitbeh - PO Box: 146404 Mazraa, Beirut, Lebanon. \\ ${ }^{3}$ Aix-Marseille Université, CNRS, CINaM UMR 7325, Campus de Luminy, Case 913, 163, \\ Avenue de Luminy, 13288 Marseille Cedex 09, France.
}

\begin{abstract}
The formation of two-dimensional electron gases (2DEGs) at $A l_{x} G a_{1-x} N / G a N$ hexagonal doublebarriers (DB) resonant tunneling diodes (RTD) is investigated by numerical self-consistent (SC) solutions of the coupled Schrödinger and Poisson equations. Spontaneous and piezoelectric effects across the material interfaces are rigorously taken into account. Conduction band profiles, band edges and corresponding envelope functions are calculated in the $A l_{x} G a_{1-x} N / G a N$ structures and likened to those where no polarization effects are included. The combined effect of the polarization-induced bound charge and conduction band offsets between the hexagonal $A l G a N$ and $G a N$ results in the formation of 2DEGs on one side of the DB and a depletion region on the other side. Using the transfer matrix formalism, the vertical transport ( $J-V$ characteristics) in $A l G a N / G a N$ RTDs is calculated with a fully SC calculation in the ballistic regime. Compared to standard calculations where the voltage drop along the structure is supposed to be linear, the SC method leads to strong quantitative changes in the $J-V$ characteristics showing that the applied electric field varies significantly in the active region of the structure. The influences of the aluminum composition and the GaN ( $A l G a N)$ thickness layers on the evolution of the current characteristics are also self-consistently investigated and discussed. We show that the electrical characteristics are very sensitive to the potential barrier due to the interplay between the potential symmetry and the barrier height and width. More interestingly, we demonstrate that the figures of merit namely the peak-to-valley ratio (PVR) of GaN / AlGaN RTDs can be optimized by increasing the quantum well width.
\end{abstract}

Keywords: Wurtzite Nitrides, Resonant Tunneling Diodes, Self-Consistent calculations, Ballistic transport.

\section{Contact author: bhouri_amel@yahoo.fr}




\section{INTRODUCTION}

III-Nitrides are one of the most interesting semiconductors for the microelectronics industry. They are the materials of choice for ultraviolet blue-green optoelectronics and they hold the potential to complement and even challenge silicon in a number of electronic applications [1]. The main reason for this enthusiasm in $(\mathrm{Al}, \mathrm{Ga}, \mathrm{In}) \mathrm{N}$ compounds stems from their direct band gap tunable along a wide range of energies. In this context, $A l G a N$ / $G a N$ heterostructures are good candidates for high power and high frequency amplifying devices for communication. Besides, due to the high energy of their longitudinal optical phonon modes (around $92 \mathrm{meV}$ in $G a N$ ), nitride semiconductors should enable the realization of quantum cascade lasers (QCLs) operating at room temperature in the $\mathrm{THz}$ frequency range.

Recent advances in digital and analog systems demand very high performance electronic circuits. Resonant tunneling diodes (RTDs) which display a Negative Differential Resistance (NDR) present very attractive characteristics [2] such as high intrinsic cut-off frequency (in the THz range). The RTD specificities are exploited in digital applications ("multi-value" logic) as well as in analog applications (ADC, frequency divider or multiplier, oscillator), leading to simpler circuits, with a large gain in power consumption and high frequency performance $[3,4]$. Thin barrier RTDs are specially promising for high frequency generation due to their extremely high speed transport properties with characteristics in order of a few picoseconds [5]. Due to their large conduction band offset (1.75 eV between $A l N$ and $G a N$ ) [6], GaN based RTDs should permit to obtain higher voltage and power operation in comparison with conventional III-V semiconductors. However, the wurtzite $A l_{x} G a_{1-x} N / G a N$ heterostructures possess strong polarization charges at the heterointerfaces making difficult the modeling of vertical electronic transport in RTDs devices. Only limited theoretical studies have been performed on $A l_{x} G a_{1-x} N / G a N$ RTDs [6-8]. Moreover, the reported electronic characteristics, principally based on the linear approximation, differ in several respects and therefore are still a topic of current discussions. There has been very few of articles using SC method in calculating RTD electronic characteristics and to our knowledge no Paper has quantitatively addressed the differences between the two methods in the GaN based RTDs as it will be presented in this work. 
The process proposed in this work goes a step further and treat with SC method the calculation of the current density across the device structure under non equilibrium conditions. Indeed, the more quantitative approach would be to consider the external applied field initially in the Hamiltonian system for SC Schrödinger-Poisson simulations and thus for a given bias, the current is determined in a SC manner.

In the present work, we propose to calculate in detail and self-consistently the free electron distribution, the conduction band profiles and the ballistic transport in $A l_{x} G a_{1-x} N / G a N$ based RTDs at room temperature. For this reason, we have developed a computer code to calculate the electrical properties of $A l_{x} G a_{1-x} N / G a N$ heterostructures. The electronic subband structures are determined by solving the coupled Schrödinger and Poisson differential equations self consistently via finite differential method [9]. We employ a proper discretization of a non-uniform grid, and taking into account the strong piezoelectric and spontaneous polarization fields exhibited by the wurtzite III-nitride heterostructures. The $J-V$ characteristics are calculated using the transfer matrix formalism assuming a ballistic transport [6]. The effects of the barrier height and internal field on electrical properties of $A l_{x} G a_{1-x} N / G a N$ RTDs are investigated by varying the Aluminum composition. The influences of crystal layout ( $G a N$ well width and $A l G a N$ barrier thickness) on the current density are also analyzed. A detailed presentation of the employed theoretical model is given in section 2 . The results of the band structure and current density calculations are presented in section 3 . Finally section 4 concludes the present work.

\section{THEORETICAL FORMULATION}

An accurate simulation of resonant tunneling diodes is of primary importance to develop reliable design tools. The RTD structure is treated as an open system and is generally divided into large reservoirs and an active region. The reservoirs model the exchange of electrons with the external electrical circuit. The active region, where the important physical parameters take place, is a short device domain consisting in a single well embedded between two barriers. Thus, numerical calculations should be investigated in such small region. Several items contribute to the high nonlinearity of the device [10] making the simulation of RTDs a delicate assignment. 


\section{2-1 Band scheme modeling}

To calculate the free-electron distribution and the conduction band profiles we employ the effective-mass approximation. Along the growth direction of the heterostructures (z-axis), the vertical quantum transport requires a SC solution of the coupled one-dimensional Schrodinger and Poisson equations. Within the effective mass theory, the Schrodinger equation is expressed as:

$\left[-\frac{\hbar^{2}}{2} \frac{\partial}{\partial z} \frac{1}{m(z)} \frac{\partial}{\partial z}+\left(e V(z)+\Delta E_{c}(z)+e V_{x c}(z)\right)\right] \phi(z)=E \phi(z)$

where $m(z)$ is the position-dependent electron effective mass in the $z$ direction, $V(z)$ is the electrostatic potential and $\Delta E_{c}(z)$ is a stepwise function due to the conduction band discontinuity. $V_{x c}(z)$ is the local exchange-correlation potential which is discarded in the present investigation because of its minor influence on the results.

The electrostatic potential $V(z)$ is determined by solving Poisson equation in the presence of polarization induced electric field given by:

$$
\frac{\partial}{\partial z}\left(-\varepsilon(z) \frac{\partial}{\partial z} V(z)+P_{t o t}(z)\right)=q\left(N_{D}(z)-n(z)\right)
$$

where $\varepsilon(z)$ is the position-dependent dielectric constant, $N_{D}(z)$ is the ionized donor doping concentration, $n(z)$ is the free-electron concentration and $P_{t o t}(z)$ is the total polarization. The other symbols are used in their conventional meanings. We note that in general, in the absence of an extrinsic electric field, the total macroscopic polarization $P_{t o t}(z)$ is given by

$$
P_{t o t}(z)=P_{z}+P_{s}
$$

where $P_{z}$ designates the strain-induced piezoelectric polarization resulting from the lattice mismatch at the interface between two materials and $P_{s}$ is the spontaneous polarization at zero strain which is recognized to be non-zero and large in wurtzite nitride compounds. 
To solve the system of equations, the finite difference method has been applied with the boundary conditions, $(\varphi(z=0)=\varphi(z=L)=0)$ at the two extremities of the studied system. The one dimension (1D) real space along the studied structure has been divided into discrete non uniform mesh points and the latter equations have been written within these discrete spacing in the active area and semi-infinite contacts. A SC procedure has been set up, where the potential $V(z)$ is obtained by solving equation (2) from an initial guess of the charge concentration, and then inserted into the Schrödinger equation. The latter equation is then solved to provide energy levels and envelope functions of the studied system. The envelope function $\varphi(z)$ is normalized over a suitable length $L$ of the structure along the z-axis such that:

$\int_{0}^{L} d z|\varphi(z)|^{2}=1$

Once the Eigen-states have been calculated, the new electron charge density is then calculated by applying Fermi Statistics:

$$
f\left(E_{i}\right)=\frac{1}{\left(1+\exp \left(\frac{E_{i}-E_{F}}{K_{B} T}\right)\right)}
$$

here, $E_{i}$ is the energy of the $\mathrm{i}^{\text {th }}$ quantized level, $T$ is the temperature, $K_{B}$ is Boltzmann constant and $E_{F}$ is the Fermi level obtained by solving the neutrality equation:

$$
\frac{m k_{B} T}{\pi \hbar^{2}} \sum_{i} \operatorname{Ln}\left(1+\exp \left(\frac{E_{i}-E_{F}}{K_{B} T}\right)\right)=\int_{-\infty}^{+\infty} N_{D}(z) d z
$$

A linear combination of the new and old values of electron charge density is then plugged into Poisson equation and the iteration repeated until convergence is achieved.

\section{2-2 Model of electronic transport}

Particles injected from the left reservoirs have a probability $T(E)$ to pass through the double barrier and to reach the right contact. If during their motions, particles maintain their phase coherence, tunneling is called coherent. In this case several approaches have been proposed to 
calculate the tunneling transport in RTDs, namely: Green function, WKB approximation and the transfer matrix formalism. In this work we have adopted the latter formalism which can be adapted to any arbitrary potential energy profile. The transfer matrix formalism [11] consists in approximating the potential $V(z)$ by a series of small potential step $V_{i}\left(z_{i}\right)$. For each section $\mathrm{i}$, the envelope function can be expressed in plane-wave form as:

$\varphi_{i}\left(z_{i}\right)=A_{i} e^{\left(j k_{i} z_{i}\right)}+B_{i} e^{\left(-j k_{i} z_{i}\right)}$

where $A_{i}\left(B_{i}\right)$ is the transmitted (reflected) amplitude and $k_{i}=\sqrt{\frac{2 m_{i}\left(E-V_{i}\right)}{\hbar^{2}}}$ is the wave vector. $A_{i}$ and $B_{i}$ are found by matching $\varphi_{i}$ and $\frac{1}{m_{i}} \frac{\partial \varphi_{i}}{\partial z_{i}}$ at each i-interface. So, for each boundary the envelope function coefficients can be related by the transfer matrix equation:

$$
\left(\begin{array}{c}
A_{i+1} \\
B_{i+1}
\end{array}\right)=M_{i}\left(\begin{array}{c}
A_{i} \\
B_{i}
\end{array}\right)
$$

where:

$$
M_{i}=\frac{1}{2}\left(\begin{array}{ll}
\left(1+\frac{m_{i+1}}{m_{i}} \frac{k_{i}}{k_{i+1}}\right) e^{j\left(k_{i}-k_{i+1}\right) z_{i}} & \left(1-\frac{m_{i+1}}{m_{i}} \frac{k_{i}}{k_{i+1}}\right) e^{-j\left(k_{i}+k_{i+1}\right) z_{i}} \\
\left(1-\frac{m_{i+1}}{m_{i}} \frac{k_{i}}{k_{i+1}}\right) e^{j\left(k_{i}+k_{i+1}\right) z_{i}} & \left(1+\frac{m_{i+1}}{m_{i}} \frac{k_{i}}{k_{i+1}}\right) e^{-j\left(k_{i}-k_{i+1}\right) z_{i}}
\end{array}\right)
$$

The relation can propagate from interface to interface. That is, we find:

$$
\left(\begin{array}{l}
A_{N} \\
B_{N}
\end{array}\right)=\prod_{i=N-1}^{1} M_{i}\left(\begin{array}{c}
A_{0} \\
B_{0}
\end{array}\right)
$$

$\left(A_{N}, B_{N}\right)$ and $\left(A_{0}, B_{0}\right)$ are the coefficients of the envelope function corresponding to collector and emitter region respectively.

The transmission probability is given by the ratio between the transmitted flux and incident flux as: 
$T(E)=\frac{\left|A_{N}\right|^{2} k_{N} / m_{N}}{\left|A_{0}\right|^{2} k_{0} / m_{0}}$

Following the Tsu-Esaki formalism [12], the current density is expressed as a function of the applied bias after integration over the longitudinal energy:

$J\left(V_{a}\right)=\left(e m k_{B} T / 2 \pi^{2} \hbar^{3}\right) \times \int_{0}^{\infty} T(E) \operatorname{Ln}\left(\frac{1+\exp \left(\left(E_{F}-E\right) / k_{B} T\right)}{1+\exp \left(\left(E_{F}-E-e V_{a}\right) / k_{B} T\right)}\right) d E$

where $V_{a}$ is the applied voltage across the active region, and the transmission coefficient $T(E)$ is calculated for the specific value of $V_{a}$. The bias will be applied on the top contact, while the bottom contact will be grounded. This configuration is favorable for the observation of resonant current as shown in Ref [6].

\section{RESULTS AND DISCUSSIONS}

\section{3-1 Parameters}

The resonant tunneling diode under investigation consists of an active region sandwiched between two $95 \mathrm{~nm}$ thick top and bottom n-doped $G a N$ contact layers with $N_{D}=5 \times 10^{18} \mathrm{~cm}^{-3}$. The undoped active region is composed of a $2 \mathrm{~nm}$-thick $G a N$ quantum well (QW) bordered by two $1 \mathrm{~nm}$-thick $A l_{x} G a_{1-x} N$ barriers. The aluminum composition, $x$, varies from 0.1 to 0.5 . This stack of layers is separated from the contact layers by $5 \mathrm{~nm}$-thick undoped $\mathrm{GaN}$ spacers. Due to its nanoscale size, the active area is assumed to be a ballistic region connected to two semi-infinite contacts [13]. The effective electron masses and the other relevant parameters for $G a N$ and $A l N$ are summarized in table-1 and taken from references [14-20]. The $A l_{x} G a_{1-x} N$ spontaneous polarization has been obtained by linear interpolation of the binary $(A l N, G a N)$ compound values.

\section{3-2 Stationary regime of unbiased RTDs}

On the basis of equations (1-6), SC calculations of the conduction band profile and band edges of the n-type designed $A l_{x} G a_{1-x} N / G a N$ RTDs are performed with no external applied bias. We display in figure 1 the $A l_{0.5} G a_{0.5} N / G a N$ conduction band profiles, the energy 
levels and their corresponding envelope functions with and without considering the effect of the total polarization. The zero energy level was arbitrarily placed at the bottom of the conduction band on the left contact and the energy Fermi level has been calculated to be close to $0.04 \mathrm{eV}$. Comparing the two curves, one can see that the effect of the two polarization components on the morphology of the structure is very noticeable mainly the formation of a triangular confinement potentials. Indeed, the electric fields are non-zero in the well and in the barriers and have opposite signs leading to an effectively triangular confinement potential, which would favor the transfer of confined electrons into the barrier layer on one side of the GaN well [21]. Peculiarly, the spontaneous and piezoelectric polarizations induce a strong asymmetry in the conduction band profile despite symmetrical barrier geometry. This finding agrees well with results of references $[6,7]$.

In $A l G a N / G a N$ heterostructures, the two components of the polarization (piezoelectric and spontaneous) are present. The polarization difference between the materials $G a N$ and $A l G a N$ induces a positive charge at the $A l G a N / G a N$ interface. Therefore, free electrons are attracted by this positive charge and tend to accumulate in a quantum well close to the interface. The internal electric fields associated with these polarizations lead to the formation of a two dimensional electron gas (2DEG) specified by a shallow triangular shaped well within the left reservoir region and a depletion region on the right side of the active region. Electrons accumulate mostly in the 2DEG of lowest energy [6]. Therefore, the 2DEG falls below the Fermi level $E_{F}$.

In table 2, we provide the calculated heterostructure Fermi level positions and the values of the energy levels confined in the $G a N$ quantum well of the studied $A l_{x} G a_{1-x} N / G a N$ RTD structures $(0.1 \leq x \leq 0.5)$ at equilibrium at $300 K$. Dashes in the table indicate that no confined levels are observed. It can be noted that the GaN QWs exhibit one $(0.1 \leq x \leq 0.3)$ or two confined levels which none of them is filled at zero bias. Moreover, as $x$ augments, the fundamental $\left(e_{1}\right)$ and the first exited $\left(e_{2}\right)$ confined levels are blue-shifted. The inset of figure 1 displays the conduction band profiles investigated for the selected aluminum composition in the barriers. As $x$ augments, the conduction band offset between $G a N$ and $A l_{x} G a_{1-x} N$ increase as expected reaching a value of about $0.8 \mathrm{eV}$ for $x=0.5$ [6] and the 
induced electric field takes stronger values. Such properties should alter the position of the confined energy levels and the envelope functions distribution.

We have calculated the electron density $n(z)$ of the $A l_{x} G a_{1-x} N / G a N$ double barrier structure for the selected aluminum compositions $(0.1 \leq x \leq 0.5)$. The simulated results are displayed in figure 2. One can note: (i) an important electron concentration is confined in the left hand reservoir in contrary to the right hand reservoir; (ii) the active region is free of electrons. As expected, the electron density in the left contact layer is systematically higher in comparison to the right contact region. As we have mentioned above, the high electric field induced by the interface charge favors the electron buildup in the left contact layer. Increasing the Al content in the barrier layers induces a large polarization charge at the GaN / AlGaN interface and therefore an increase of the electron concentration in the left channel as shown in figure 2. However, the right hand reservoir becomes further depleted with increasing aluminum composition. The density within the central quantum well is minimal indicating that the GaN central well remains practically empty and does not depend on Al composition. Thus, $A l_{x} G a_{1-x} N / G a N$ studied structure is appropriate to resonant tunneling device vitally controlled by the amount of aluminum composition in the barrier layers.

\section{3-3 Transport calculations}

Using previous mentioned equations, transmission coefficient $T(E)$ of unbiased $A l_{x} G a_{1-x} N / G a N$ double barriers single well RTDs are calculated at $T=300 K$. Figure 3 shows the comparative evolution of transmission coefficient profile with electron energy when varying aluminum composition in $A l_{x} G a_{1-x} N$ barriers. Fermi energy of $G a N$ is located at about $0.04 \mathrm{eV}$ above the conduction band minimum. As it can be noted, depending on aluminum composition, the transmission coefficient present one or two peaks: (i) The first peak is attributed to the resonant tunneling through the first quasi-bound state in the quantum well and (ii) the second peak is assigned to the resonant tunneling through the excited quasibound state or to a continuum state near the double barrier edge. The transmission probability of electrons from $G a N$ contact layer through the $A l_{x} G a_{1-x} N$ barrier decreases as $x$ increases and the maximum tunneling probability occur when the mole fraction of Aluminum is $10 \%$. Besides, the variation of transmission coefficient with electron energy under zero applied bias shows that the resonant peaks are distinctly shifted towards higher energies when increasing $x$ 
. In fact, the energy of the first quasi-bound state increases with Al composition from around $0.14 \mathrm{eV}$ for $x=0.1$ to $0.51 \mathrm{eV}$ for $x=0.5$ (see table 2). As it can be noted, no interesting features are seen above $0.45 \mathrm{eV}$ and $1.7 \mathrm{eV}$ for $x=0.1$ and $x=0.5$ respectively, where the transmission coefficient becomes very close to unity.

To explain the origin of the energy blue-shift of the transmission resonant peak when increasing aluminum composition, we consider table 2 together with the inset of figure 1 which shows different conduction band profiles calculated for several alloyed barriers. It is clearly shown that in polar heterostructures, the double-barrier potential and the energy levels depend strongly on the barrier composition. In fact, as $x$ increases from 0.1 to 0.5 , the $A l_{x} G a_{1-x} N$ band gap energy and the conduction band offsets between $G a N$ and $A l_{x} G a_{1-x} N$ increase (see table 2). Furthermore, the internal field increases when increasing $x$ in the double barrier since the polarization difference between $G a N$ and $A l_{x} G a_{1-x} N$ is highly influenced by composition [6,7]. When $x$ increases, the structure exhibits a wider depletion region on the right contact and deep well-filled inversion regions on the left side. As a result, the quantum well is pushed far from the conduction band edge in the contact layer. Such unique phenomena blue-shift and reduce the transmission resonant peak probabilities.

Numerical calculations of the $J-V$ characteristics of $A l_{x} G a_{1-x} N / G a N$ double barrier structure have been investigated to bring out the importance of SC approach and the effects of structure parameters. Indeed, for calculating the current density, we have considered two methods:

(i) The first method calculates the current density with a nonlinear treatment i.e. with a SC approach. In fact, to obtain $J-V$ characteristics, we first calculate, at zero bias, the band conduction position by solving self-consistently the coupled Schrödinger and Poisson equations. After achieving convergence, an external polarization is applied to the obtained conduction profile, assuming that the potential drop occurs linearly across the system. A new polarized conduction band potential position must be updated by recomputing it selfconsistently. Once the SC solution for a given bias is achieved, the current is then calculated on the basis of equation 12 .

(ii) The second method consists on considering an original process: we should first compute the conduction band potential for the unbiased structure with SC treatment. Then, we consider a linear voltage drop across the active region between the left spacer and the end of the 
depletion region where the semiconductors are non-degenerate. Finally we calculate the current density directly on the basis of equation 12 .

In simulating $J-V$ characteristics, we have considered the same active region defined in section 3-1.The simulated current density characteristics under an external applied bias are displayed in figure 4. Figure 4(a) presents the evolution of the current density as a function of the applied bias, calculated using the SC method and figure 4(b) illustrates the results of the non-SC calculations.

At first sight, we note that the obtained current density characteristics, computed on the basis of the two mentioned methods, present the same behaviors. For all considered Al compositions $(0.1 \leq x \leq 0.5)$, theoretical current-voltage characteristics reveal the presence of a typical negative differential resistance (NDR) region obviously associated with the constructive interference of the electron waves inside the quantum well. Under forward bias conditions, a typical peaked $J-V$ curve can be observed due to resonant tunneling through the lowest quasi-bound state. These effects can qualitatively be understood by examining the energy dependence of the central QW quasi-bound states on the external applied bias. The inset of figure 5 describes the evolution of the fundamental energy level as a function of external applied bias. At zero bias, the central QW levels of the $A l_{x} G a_{1-x} N / G a N$ RTDs $(0.1 \leq x \leq 0.5)$ are above the Fermi level indicating that the quantum wells remain unpopulated when varying aluminum composition. As the external applied bias increases, the resonant levels are progressively red-shifted and can be aligned with the Fermi level. As a result, resonant tunneling can be revealed leading to a 3D-2D electron transfer. In fact, at resonance, the electron like-wave can easily tunnel through the $2 \mathrm{D}$ region between the barriers. In the QW, the electron like-wave will oscillate back and forth until reaching a stationary state and then leak into the continuum in the collector side. Beyond resonance, the transport is sequential i.e. the transmission probability of electrons through each potential barrier is independent. Therefore, electrons have a small probability to travel into the well and a sharp drop in the current occurs. As the voltage drop across the active region increases, the valley current will be enhanced. Moreover, from figure 4, it is clear that the resonant current peaks occur at a much higher applied bias when Al content in the barriers increases and therefore sharply blue-shifts the quasi-bound states relative to the Fermi level. The $300 \mathrm{~K}$ obtained results for all considered $\mathrm{Al}$ composition are given in table 3. 
Next, we compare the $J-V$ characteristics obtained calculated with and without SC effects. It can be seen from figure 4(a) and 4(b) that, within the SC model and for all considered aluminum compositions, both peak and valley currents are significantly decreased, and the current peak occurs at higher applied bias.

In order to evaluate the existence of such dissimilarities between the obtained results, we present in figure 5 the potential profile of the $A l_{0.5} G a_{0.5} N / G a N$ RTD, calculated at selected applied bias based self- (solid line) and non-self- (dashed line) consistent methods. As can be noted within SC calculations, the central QW and the depletion region energy positions are shifted to higher energies. Therefore, for all the considered applied bias, the SC-potential raises the resonant level by about $100 \mathrm{meV}$ relative to the linear approximation. Such effects shift the current peak to even higher applied bias.

In the comparison of the current levels, the potential profiles should be studied in further details. As it can be noticed from Fig (5), in the SC calculations, the potential is lowered in the 2DEG layer with respect to the linear approximation due to the high electron density in this region. This increase of the electron density is a consequence of interference effects at the interface between the 2DEG and the left barrier. Hence the injected electrons will have a high probability to be captured in the ground state of 2DEG before tunneling. As a result of the accumulation of electrons in this area, the potential energy will be red-shifted in the 2DEG and blue-shifted in the depletion region with respect to the linear calculations. Therefore, the probability of one electron to tunnel resonantly through the active region is lowered in the SC profile due to the high degree of asymmetry of the potential profile [22]. Therefore, the collected current will be decreased.

Although the valley and peak currents are seemingly reduced within SC calculations, the current pic-to-valley exhibits an enhancement compared to the linear calculations which is mainly due to the presence of a wider depletion region responsible of the suppression of the leakage (valley) current.

Under external bias, the effect of the SC method on $J-V$ the characteristics is significant. In fact, the SC model is well adapted and suitable for handling boundary effects and taking into account impacts of space charges in order to fold the influence of the left and right semiinfinite leads into the active area. According to our simulated results, it is clear that the response of the contacts serving as charge reservoirs must be included into any meaningful 
SC calculations. Indeed, within the linear approach, we have considered rigid boundary condition and linear potential drop across the double barrier structure. However, these conditions cannot exclusively establish the electronic characteristics of the structure. Therefore, it is required that the injection of particles at the contacts should be adjusted in a SC way to meet charge neutrality in the asymptotic regions and constant current density throughout the circuit.

\subsection{Effect of crystal layout}

Several parameters are important for improving the performance of the RTD structures for example the thickness and the height of the well and barrier layers. The following point addressed in this paper focuses on analyzing the influence of the height and thickness layers on the $J-V$ characteristics of the double barrier $A l_{x} G a_{1-x} N / G a N$ structures.

It was observed in figure 3 that the aluminum mole fraction significantly affects the position of the transmission resonant peaks and their intensities. In fact, when rising $x$, the transmission probabilities are lowered and their corresponding peaks become deep and sharp and shift progressively to higher energies. These findings can be justified by the following: firstly the conduction band offset in $A l_{x} G a_{1-x} N / G a N$ strained systems is function of aluminum composition and increases when increasing $x[6,7]$ and secondly the internal field is dependent on the structure geometry and change with barrier heights [7]. Indeed as mentioned in table 2, it is blue-shifted relatively to the Fermi level when aluminum content augments in the barrier which explains well the behaviors of the transmission probabilities. Furthermore, the current peak progressively shifts to higher voltages and is slowly reduced when increasing Al content from 0.1 to 0.5 . The valley current is strongly reduced and therefore enhancing the PVR.

In this paragraph, we will consider the influence of the barrier thickness $\left(L_{b}\right)$ (figure 6(a)) and quantum well width $\left(L_{w}\right)$ (figure 6(b)) on the $J-V$ characteristics of $A l_{0.5} G a_{0.5} N / G a N$ RTD.

Figure 6(a) illustrates the dependence of the SC simulated current density on the barrier width $L_{b}\left(0.5 \leq L_{b} \leq 2 \mathrm{~nm}\right)$. The thickness of the GaN quantum well is taken constant equal to $2 \mathrm{~nm}$. We find that $\mathrm{J}-\mathrm{V}$ curves sensitively response to the modification of the size barriers. As 
expected, the peak and valley currents decrease and the PVR is strongly enhanced as the barriers are becoming larger [7]. At $300 K$, the PVR exhibits a maximum of 46 for $2 \mathrm{~nm}$ barriers and a minimum of 7 when $L_{b}=0.5 \mathrm{~nm}$. Moreover, when $L_{b}$ increases from 0.5 to 2 $\mathrm{nm}$, the current peak occurs at higher bias. To discuss the origin of such findings, we consider the inset of figure 6(a) illustrating the calculated conduction band profiles for several barrier widths. It is clear that the energy position of both well and barriers are strongly dependent on barrier thickness $L_{b}$ which is a fundamental parameter for controlling the internal electric field in wurtzite heterostructures [23]. Indeed, the internal electric field modifies the potential shape: the central QW resonant levels are pushed far from the conduction band edge in the contact layers and the depletion region becomes larger. These effects appear strong and lead to a reduction in the current peak intensities and an enhancement of the PVR. However, a large PVR drops if the current peak is too low which is the case when we consider the largest barrier $L_{b}=2 \mathrm{~nm}$. There is no clear tendency of the PVR with the barrier width. This figure of merit of RTD is a function of two competing mechanisms: the current peak that depends on the symmetry of the DB potential and the valley current that decreases with the barrier height and width. A compromise between the symmetry and the barrier composition should be found in the optimization of the PVR.

In the following, the $A l_{0.5} G a_{0.5} N / G a N$ current-voltage characteristics are discussed with respect to the $G a N$ QW width. All combinations between 0.5 and $2 \mathrm{~nm}$ for the GaN QW thicknesses are simulated by maintaining the AlN barriers' thicknesses at $1 \mathrm{~nm}$. The SC calculations of the conduction band potential for each of these structures show that a single quantum level may be confined in the well with $G a N$ thickness: 0.5, 1 and $1.5 \mathrm{~nm}$. By increasing the thickness of the well, this level becomes deeper and second quantum level confines in the well for a thickness equal to $2 \mathrm{~nm}$ (see the inset of figure 6(b)). Numerical calculations of the $J-V$ characteristics are given in figure 6(b). As one can note, the resonant peak shifts promptly to lower voltage as the thickness of the well increases. This effect can be qualitatively understood by looking at the dependence of the confined quasi-bound state on the well width displayed in the inset of figure $6(\mathrm{~b})$. As $L_{w}$ increases the resonant and the Fermi levels become closest requiring less external bias to achieve resonance. From the same figure we can also see a quasi-linear decrease in the peak and valley current intensities due to the decrease of the electrons probability transmission. The valley current is strongly reduced however the current peak decreases slowly inducing a strongly enhancement of the PVR, 
from 5 for $0.5 \mathrm{~nm}$ well width to 38.4 for $2 \mathrm{~nm}$ well width. These observations are very promising for the following reason: there is a clear tendency of the PVR and the electrical power with the well width. When the well becomes larger, the PVR increases and both the resonant voltage and the current peak decrease. Therefore, it is more straightforward to design an optimized DTR structure for a fixed content of the DB. Finally, we should point out that there is an upper limit of the quantum well width determined by the position of the energy level inside the well relative to the Fermi level in the emitter. The former should be always lower in energy so that resonant tunneling can arise.

\subsection{Discussion}

In the previous paragraphs, we have shown that in calculating electronic characteristics of such resonant tunneling diodes, and for better accuracy, one has to solve, for the whole structure (active region and contacts), the coupled Poisson- Schrödinger equations for each given applied bias. For materials like $\mathrm{GaN}$, which exhibits an internal polarization, one has to consider the effect of the space-charge distribution in the whole structure. Indeed, with SC calculations, the space charge layers that appear both near the contact regions and in the electron populated quantum well are taken into account which will result in accurate quantitative estimation of the $J-V$ characteristics of the RTDs.

Furthermore, the PVR and the NDR are the two important factors for determining, the performance of RTDs. These factors depend on several parameters such as barrier height, well width, asymmetric profile, contact doping concentration.... There have been experimental and theoretical attempts to evaluate the dependence of these factors on such parameters. For selected RTD structures, Table 4 shows our calculated PVR values along with some theoretical and experimental data available in the literature for comparison.

As it can be seen, there are still large uncertainties in the reported current peak to valley (GaN/AlGaN) RTDs. The authors of references [6, 7] have theoretically investigated the $J-V$ characteristics of $\mathrm{GaN}$ based RTDs and reported that these characteristics are significantly affected by the conduction band asymmetry and the geometrical parameters. Such results are in agreement with our findings. Moreover our obtained PVR values differ slightly from the theoretical available ones.

Besides the previously cited parameters, the authors of reference [24, 28, 29, 31], reported that $\mathrm{AlGaN} / \mathrm{GaN}$ RTD experimental characteristics are also dependent on device size and substrate choice. In fact, they showed that lateral epitaxial overgrowth GaN (LEO) on 
sapphire substrate is the most preferable template that can be used to get higher PVR [29]. The authors of reference [25] reported that they have observed, only in the small area devices, an exactly reproducible NDR signature for low-aluminum $\mathrm{AlGaN} / \mathrm{GaN}$ double barrier RTD structures and suggest that charge transport is affected by leakage paths through dislocations. Sakr et al. [26] have demonstrated that the current voltage measurements of GaN baser RTDs show, at room temperature, reproducible NDR and a current hysteresis that disappear when decreasing the temperature. Thus, the latter authors propose that the room temperature NDR is not due to electron resonant tunneling but related to trapping effect.

We claimed that the discrepancies between our results and the published ones arise from the numerical parameter values used in our calculations and the distinct techniques of investigating conduction band profiles and current voltage characteristics. Therefore, we expect that more experimental data are necessary to confirm precisely RTD parameters which still have some uncertainties.

\section{CONCLUSIONS}

In summary, we present numerical SC results for the dependence of the conduction band profiles on the aluminum composition $(0.1 \leq x \leq 0.5)$ in a doped wurtzite $A l_{x} G a_{1-x} N / G a N$ based RTD, under the effects of the intrinsic polarization fields. The combined effects of polarization-induced bound charge and the conduction band offset between the hexagonal $A l_{x} G a_{1-x} N$ and $G a N$ compounds results in the formation of 2DEG and depletion region on the left and right hand contacts respectively. A substantial enhancement of the 2DEG density is revealed however the depletion region becomes further depleted for higher Al composition. Next, we calculate self-consistently the static $J-V$ characteristics for selected Al compositions. Results show the presence of Negative Differential Resistance (NDR) and an enhancement of PVR when Al composition increases. The SC simulated $J-V$ curves are then compared to those calculated applying a linear approach. The results obtained from the two methods present dissimilarities. Indeed, it is shown that (i) the responses of the contacts serving as charge reservoirs must be included in calculating the $J-V$ characteristics, (ii) with SC calculations, the charge effects decrease the peak and valley currents, nevertheless the PVR are enhanced compared to those obtained using the linear approximation. Calculating the $J-V$ curves for different barrier and well widths, we suggest that the PVR can be easily 
maximized by controlling the well width. This information can be very useful for designing devices that utilize the $J-V$ characteristics of double barrier resonant tunneling structures.

\section{REFERENCES}

[1] S. Chowdhurry and D. Biswas, International Journal of Nano Devices: Sensors and Systems. 1, 39 (2012).

[2] W. Liou and P. Roblin, Electron. Lett., 41, 1098 (1994).

[3] H. C.Lin, Proc. IEEE Int. Symp. Multiple Valued Logic (ISMVL 94), IEEE Press, 188 (1994).

[4] H.J. De Los Santos, K. Chui, D. Chow and H.1. Dunlup, IEEE Microwave Wireless Compon. Lett. 11, 193 (2001).

[5] P. H. Handel and A.G. Tournier, IEEE Proc. 93, 1784 (2005).

[6] S. Sakr, E. Warde, M. Tchernycheva, and F. H. Julien, J. Appl. Phys. 109, 023717 (2011).

[7] M. Boucherit, A. Soltani, M. Rousseau, J.-L. Farvacque and J. -C. De Jaeger, J . Appl. Phys. 112, 114305 (2012).

[8] E.Warde, S. Sakr, M. Tchernycheva, and F.H. Julien, Journal of electronic materials, 41, 965 (2012).

[9] I.H. Tan, G. L. Snider, L. D. Chang and E. L. Hu , J. Appl. Phys. 68, 4071 (1990).

[10] W. Pötz, J. Appl. Phys., 66 , 2458 (1989).

[11] J.-G. S. Demers and R. Maciejko, J. Appl. Phys. 90, 6120 (2001).

[12] R. Tsu and L. Esaki, Appl. Phys. Lett. 22, 562 (1973).

[13] A. Bhouri, A. Rached and J-L Lazzari, J. Phys. D: Appl. Phys. 48, 385102 (2015).

[14] I. Vurgaftman and J. R. Meyer, J. Appl. Phys. 89, 5815 (2001).

[15] I. Vurgaftman and J. R. Meyer, J. Appl. Phys. 94, 3675 (2003).

[16] V. Bougrov, M.E. Levinshtein, S.L. Rumyantsev, and A. Zubrilov, John Wiley \& Sons, Inc, New York Chapter 1 (2001). 
[17] B. Foutz, S. O’Leary, M. Shur, and L. Eastman, J. Appl. Phys. 85, 7727 (1999).

[18] V.A. Fonoberov et al, J. Appl. Phys. 94, 7178 (2003).

[19 ] V. W. L. Chin, T. L. Tansley, and T. Osotchan., J. Appl. Phys. 75, 7365 (1994).

[20] O. Ambacher, J. Majewski, C. Miskys, A. Link, M. Hermann, M. Eickhoff, M. Stutzmann, F. Bernardini, V. Fiorentini, V. Tilak, B. Schaff, and L. F. Eastman, J. Phys.: Condens. Matter. 14, 3399 (2002).

[21] F. Bernardini and V. Fiorentini, Phy. Stat. Sol. (b) 216, 391 (1999).

[22] F. Bernardini and V. Fiorentini, Phys. Rev. B 57, R9427 (1998).

[23] M. Leroux, N. Grandjean, J. Massies, B. Gil, P. Lefebvre, and P. Bigenwald, Phys. Rev. B 60, 1496 (1999).

[24] C. Bayram, Z. Vashaei, and M. Razeghi, Appl. Phys. Lett. 97, 181109 (2010).

[25] D. Li, L. Tang, C. Edmunds, J. Shao, G. Gardner, M. J. Manfra, and O. Malis, Appl. Phys. Lett. 100, 252105 (2012).

[26] S. Sakr, E. Warde, M. Tchernycheva, L. Rigutti, N. Isac, and F. H. Julien, Appl. Phys. Lett. 99, 142103 (2011).

[27] M. Boucherit, A. Soltani, E. Monroy,M. Rousseau, D. Deresmes, M. Berthe, C. Durand, and J.-C. De Jaeger, Appl. Phys. Lett. 99, 182109 (2011).

[28] C. Bayram, Z. Vashaei, and M. Razeghia, Appl. Phys. Lett. 96, 042103 (2010).

[29] Z. Vashaei, C. Bayram, and M. Razeghi, Appl. Phys. Lett. 107, 083505 (2010).

[30] A. Kikuchi, R. Bannai, K. Kishino, C.-M. Lee, and J.-I. Chyi, Appl. Phys. Lett. 81, 1729 (2002).

[31] C. Bayram, Z. Vashaei, and M. Razeghia, Appl. Phys. Lett. 97, 092104 (2010). 
Table 1: $G a N$ and $A l N$ physical parameters

$\begin{array}{lll}\text { Parameters } & \text { GaN }\end{array}$

$\begin{array}{ccc}\mathrm{a}(\AA) & 3,189^{13,14} & 3,112^{13,14} \\ \mathrm{c}(\AA) & 5,185^{13,14} & 4,982^{13,14} \\ \mathrm{E}_{\mathrm{g}}(\mathrm{eV}) & 3,39^{15} & 6,2^{16} \\ m_{e}^{\|} & 0,2^{13,14} & 0,32^{14} \\ \varepsilon_{\mathrm{stat}}^{\|} & 10,1^{17} & 8,5^{18} \\ \mathrm{C}_{13}(\mathrm{GPa}) & 106^{13,19} & 108^{19} \\ \mathrm{C}_{33}(\mathrm{GPa}) & & 373^{13} \\ \mathrm{e}_{31}\left(\mathrm{C} / \mathrm{m}^{2}\right) & 398^{13} & -0,5^{13} \\ \mathrm{e}_{33}\left(\mathrm{C} / \mathrm{m}^{2}\right) & -0,029^{13} & 1,79^{13} \\ \mathrm{P}_{\mathrm{sp}}\left(\mathrm{C} / \mathrm{m}^{2}\right) & 1,27^{13} & -0,081^{13}\end{array}$


Table 2: Fermi and confined energy levels values as a function of composition aluminum $x$ in the $A l_{x} G a_{1-x} N / G a N$ RTD at equilibrium at $300 K$.

\begin{tabular}{lccccc}
\hline \hline \% (Aluminum) & $\mathbf{1 0}$ & $\mathbf{2 0}$ & $\mathbf{3 0}$ & $\mathbf{4 0}$ & $\mathbf{5 0}$ \\
\hline & & & & & \\
$\Delta \mathbf{E c}(\mathbf{e V})$ & 0.106 & 0.232 & 0.378 & 0.544 & 0.730 \\
$\mathbf{E}_{\mathbf{F}}\left(\mathbf{1 0}^{-\mathbf{2}} \mathbf{e V}\right)$ & 4.16 & 4.18 & 4.22 & 4.27 & 4.33 \\
$\mathbf{E}_{\mathbf{1}}(\mathbf{e V})$ & 0.14 & 0.24 & 0.32 & 0.42 & 0.52 \\
$\mathbf{E}_{\mathbf{2}}(\mathbf{e V})$ & ----- & ---- & ---- & 0.92 & 1.06 \\
\hline \hline
\end{tabular}


Table 3: $300 K$ values of PVRs and peak positions with different Aluminum concentration in the barriers calculated by the linear approximation method (A-L) and by the SC method (S-C).

$\%$ (Aluminum)

10

20

30

40

50

$\begin{array}{llllll}\mathrm{A}-\mathrm{L} & 1.23 & 1.74 & 3.8 & 4.51 & 31.0\end{array}$

PVR

$\underline{\text { S-C }}$

1.3

3.45

4.04

4.92

38.4

$\underline{\mathrm{A}-\mathrm{L}}$

0.2

0.35

0.75

1.25

1.75

Peak Voltage (V)

$\begin{array}{llllll}\text { S-C } & 0.1 & 0.4 & 1.0 & 1.75 & 2.45\end{array}$


Table 4: PVR Values of $\mathrm{Al}_{\mathrm{x}} \mathrm{Ga}_{1-\mathrm{x}} \mathrm{N} / \mathrm{GaN}$ RTD structures.

\begin{tabular}{|c|c|c|c|c|c|c|c|c|}
\hline & & & \multicolumn{2}{|c|}{ Experimental } & \multicolumn{2}{|c|}{ Theoretical } & \multicolumn{2}{|c|}{ This work } \\
\hline$\overline{x(\mathrm{Al})}$ & $\begin{array}{c}\mathrm{L}_{\mathrm{b}} \\
(\mathrm{nm})\end{array}$ & $\begin{array}{c}\mathrm{L}_{\mathrm{w}} \\
(\mathrm{nm})\end{array}$ & ND (cm-3) & PVR & $\mathrm{ND}(\mathrm{cm}-3)$ & PVR & ND (cm-3) & PVR \\
\hline 0.1 & 1.6 & 1.6 & $3 \times 10^{19}$ & $1.08[24]$ & & & & \\
\hline 0.18 & 2.4 & 2.4 & $1 \times 10^{19}$ & $1.03[25]$ & & & & \\
\hline 0.2 & 1 & 1 & & & $5 \times 10^{18}$ & $2[6]$ & & 2.01 \\
\hline 0.3 & 1 & 2 & & & $5 \times 10^{18}$ & $6[26]$ & $5 \times 10^{18}$ & 4.04 \\
\hline 0.5 & 1 & 2 & & & $5 \times 10^{18}$ & $41[6]$ & $5 \times 10^{18}$ & 38.4 \\
\hline 0.7 & 2 & 2 & $5 \times 10^{18}$ & 2 & & & & \\
\hline 1 & $\begin{array}{l}1 \\
1 \\
1\end{array}$ & $\begin{array}{c}1 \\
2 \\
0.8 \\
\\
0.75\end{array}$ & $\begin{array}{c}5 \times 10^{19} \\
4 \times 10^{18} \\
4 \times 10^{18} \\
8 \times 10^{17}-1 \times \\
10^{19}\end{array}$ & $\begin{array}{c}3.5[27] \\
32[\mathbf{2 8}] \\
2.15-1.42 \\
{[\mathbf{2 9}]} \\
32[\mathbf{3 0}]\end{array}$ & $5 \times 10^{18}$ & 455 [6] & & \\
\hline
\end{tabular}




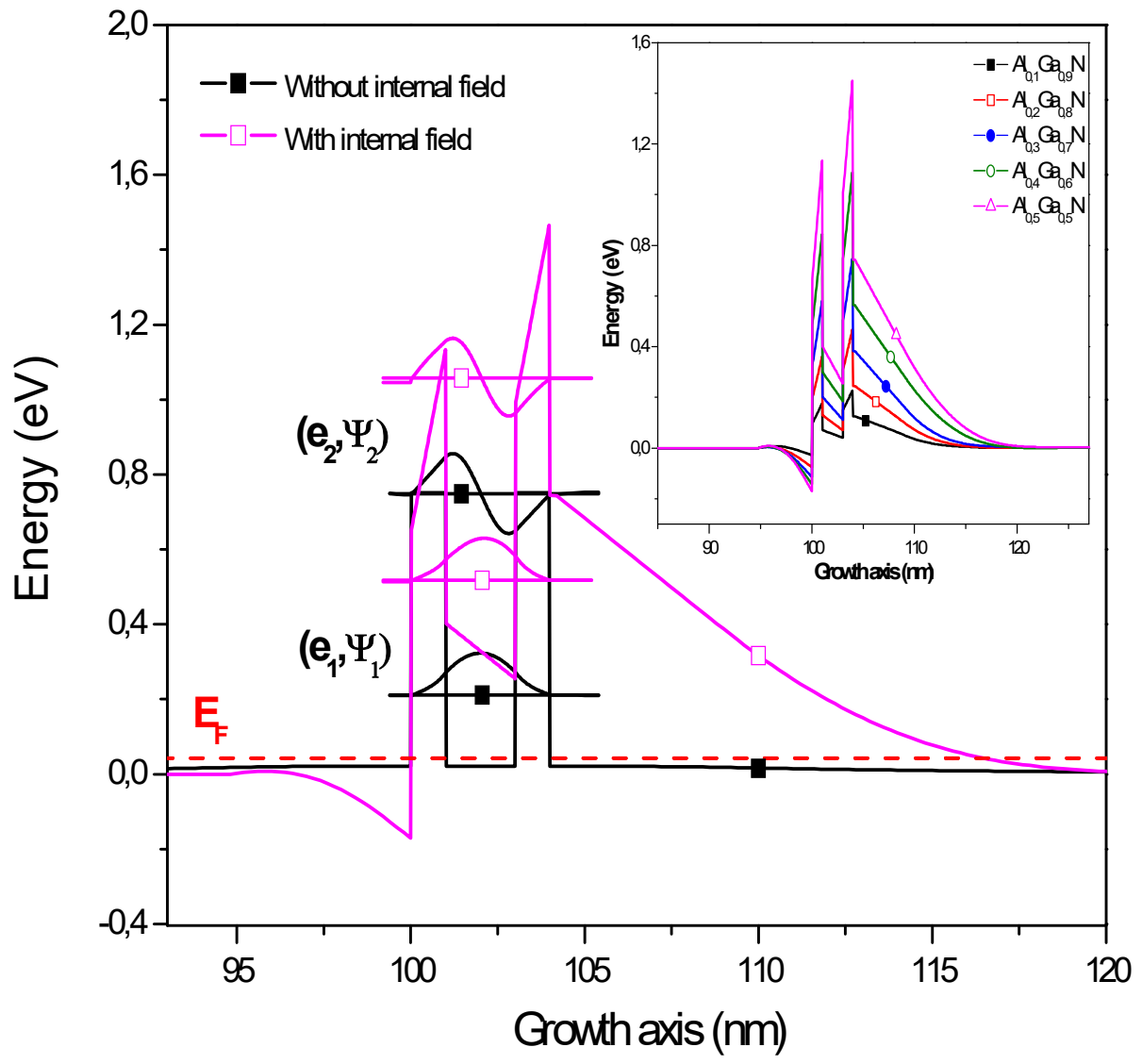

Figure1: $\mathrm{Al}_{0.5} \mathrm{Ga}_{0.5} \mathrm{~N} / \mathrm{GaN}$ conduction band profile, energy levels and their corresponding wave functions simulated with (magenta lines) and without (black lines) taking into account polarization effects. The inset plots the conduction band diagrams for different Aluminum compositions. 


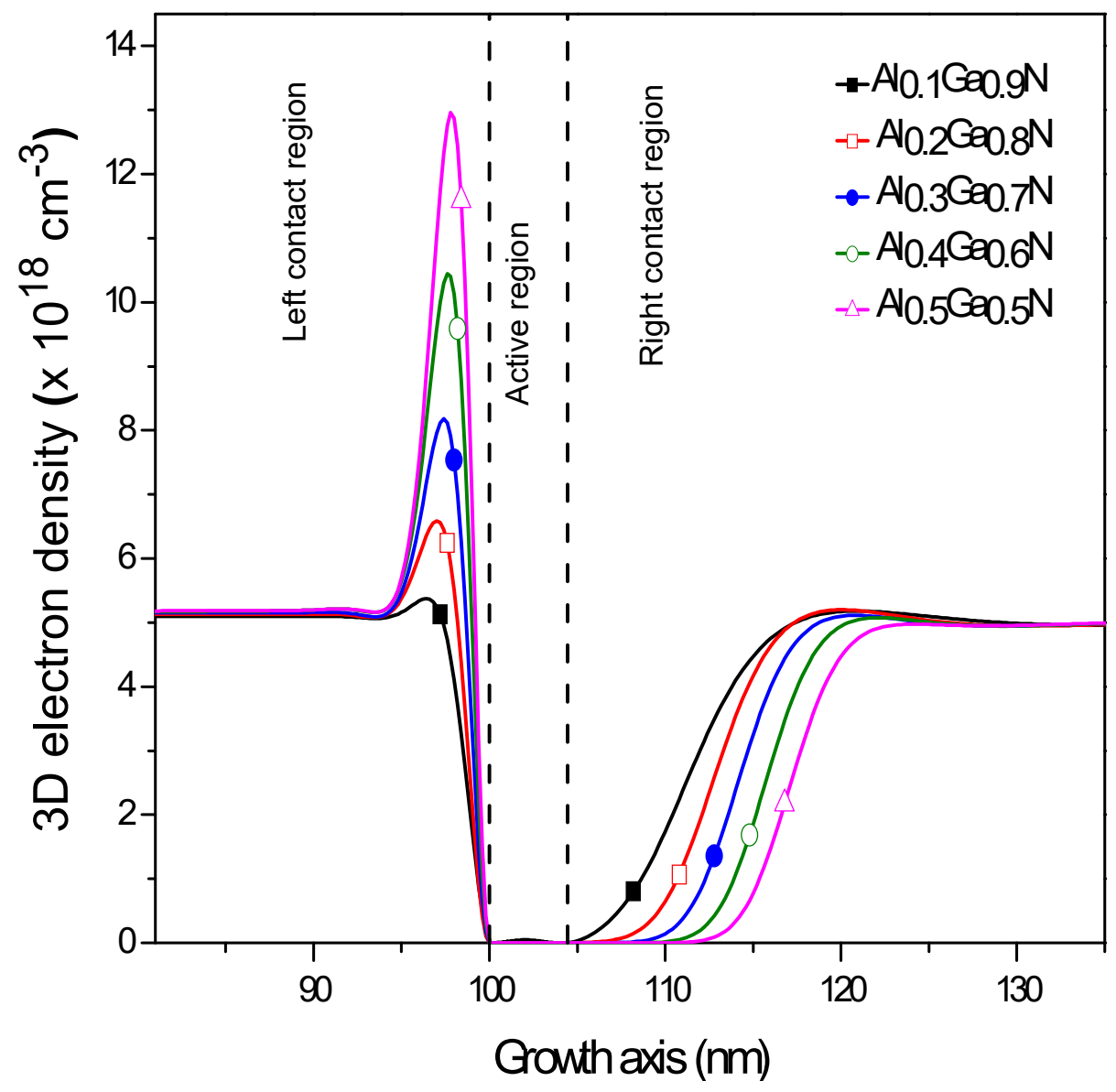

Figure 2: Electron density $n(z)$ in $A l_{x} G a_{1-x} N / G a N$ structures calculated at $300 K$. The aluminum composition is varying from 0.1 to 0.5 . 


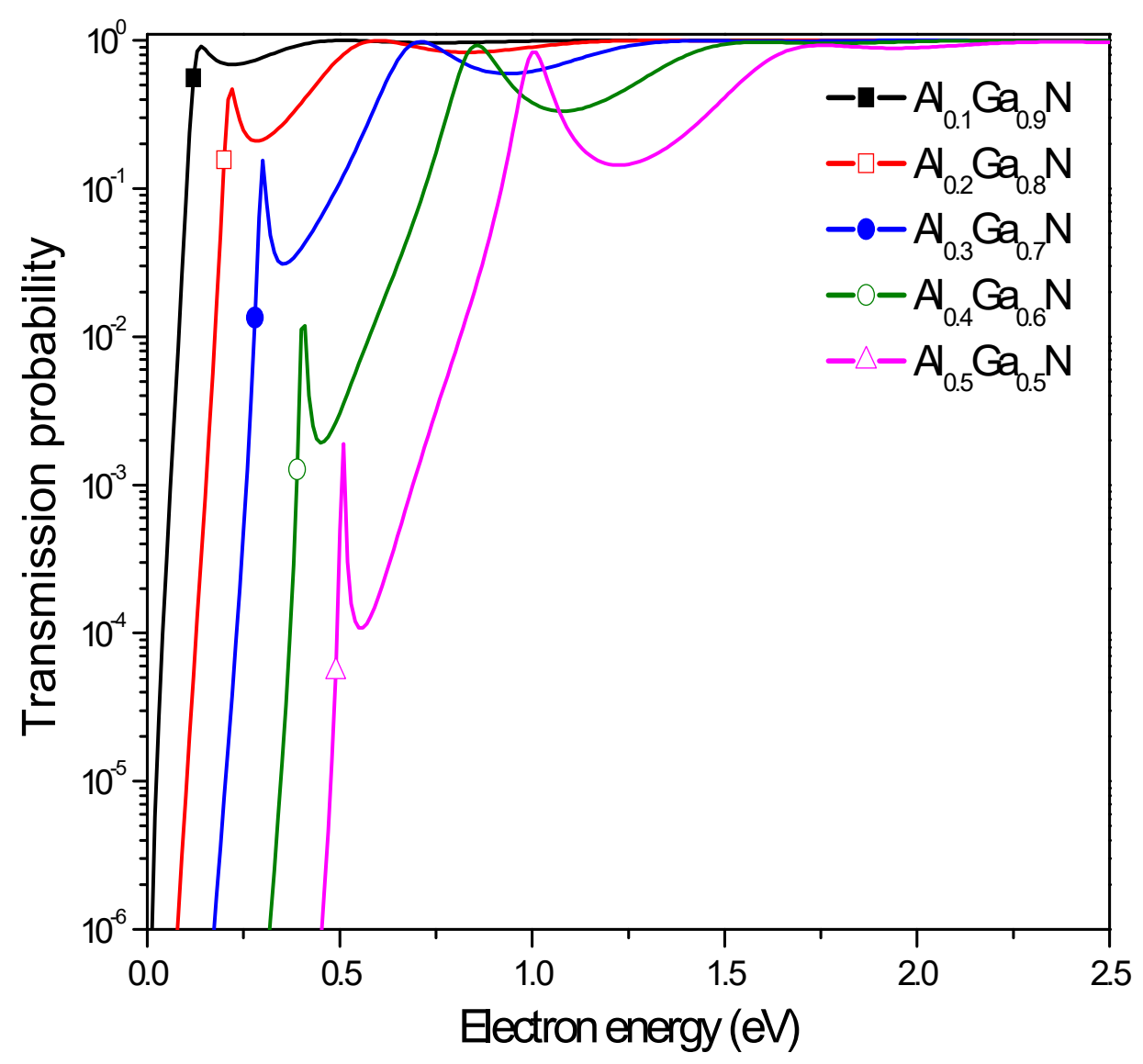

Figure 3: Comparative transmission coefficient profile for double barrier single well $A l_{x} G a_{1-x} N / G a N$ RTD under zero applied electric field calculated for different aluminum composition. 


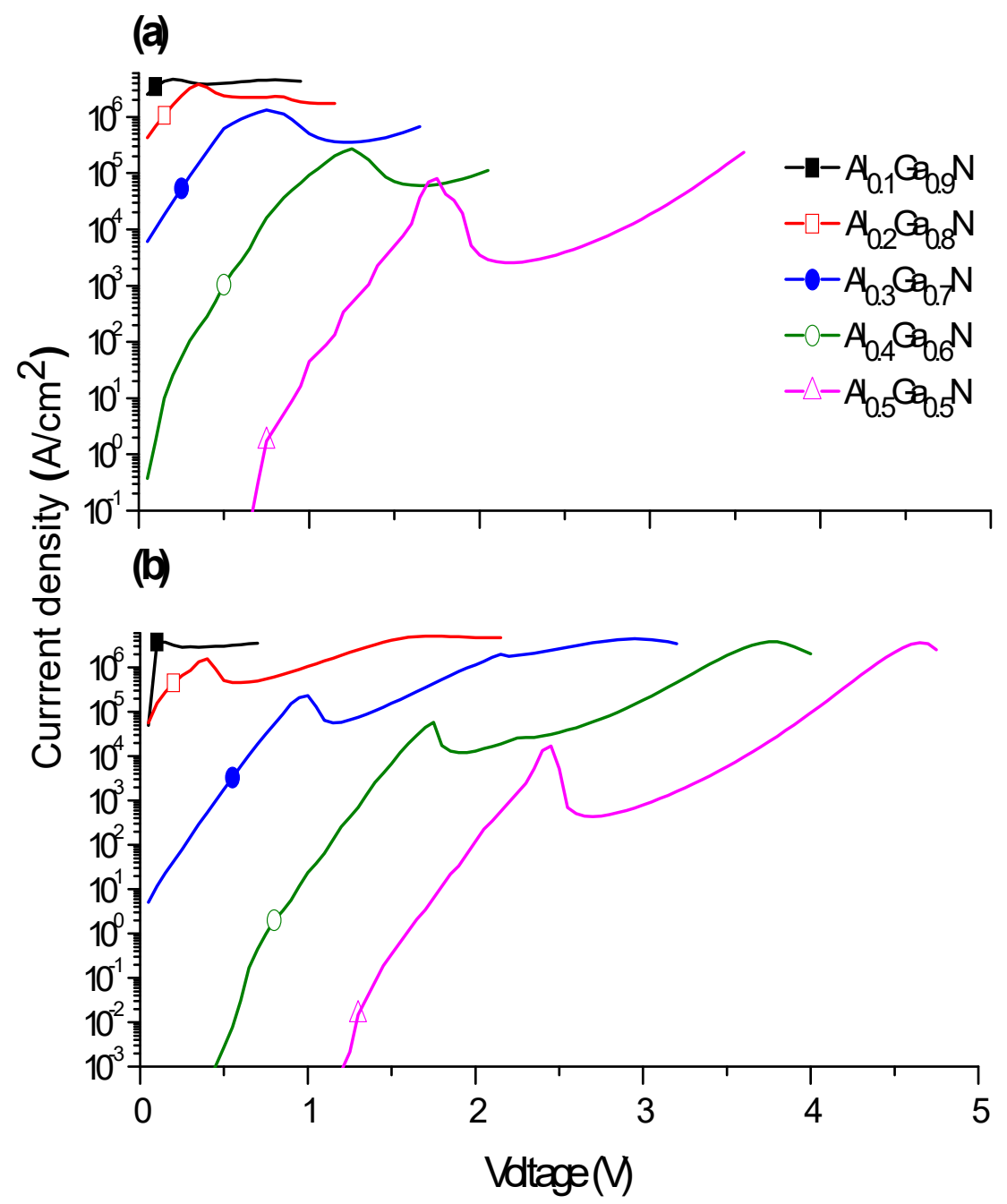

Figure 4: The $J-V$ curves of the double barrier $A l_{x} G a_{1-x} N / G a N$ structure simulated for different Al composition: (a) without SC effects and (b) including SC effects. 


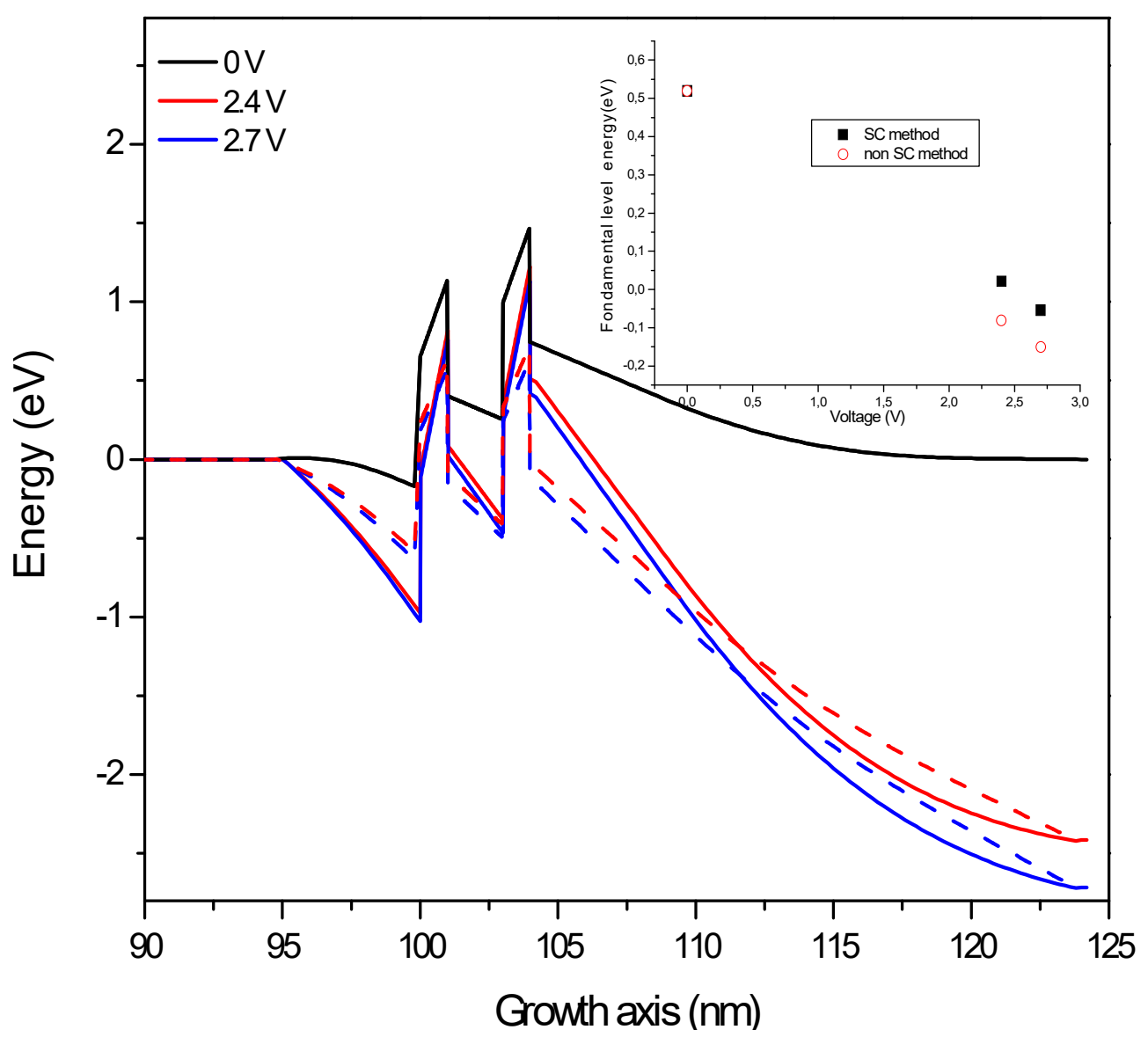

Figure 5: Conduction band profile for the $A l_{0.5} G a_{0.5} \mathrm{~N} / \mathrm{GaN}$ double barrier structure calculated for various applied bias. Solid line: SC method and dashed line: non SC method. 

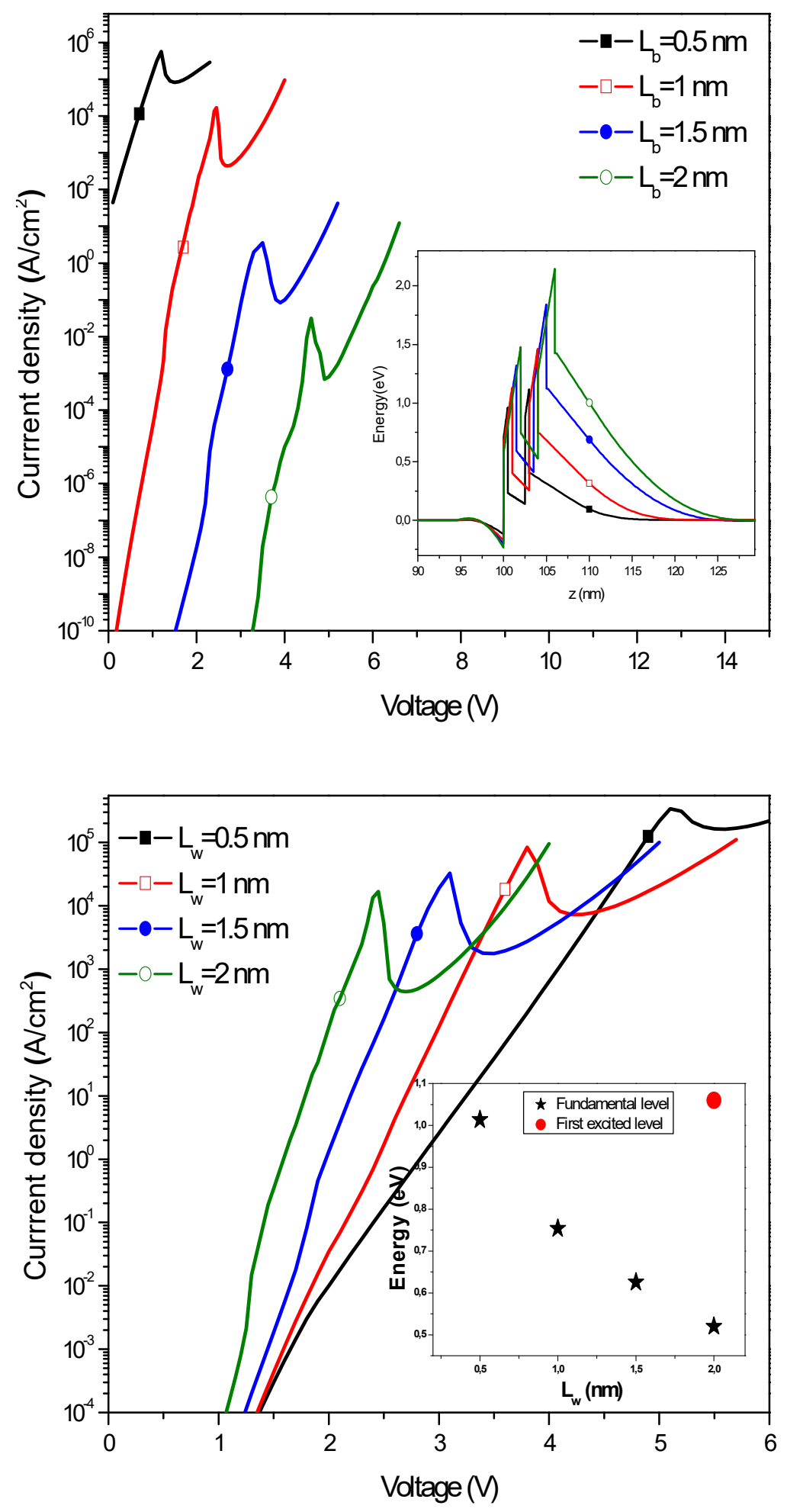

Figure 6: Current density versus external applied bias calculated self-consistently for different thicknesses: (a) of $A l G a N$ barrier layer and (b) of $G a N$ quantum well. 


\section{Figure captions}

Figure1: $\mathrm{Al}_{0.5} \mathrm{Ga}_{0.5} \mathrm{~N} / \mathrm{GaN}$ conduction band profile, energy levels and their corresponding wave functions simulated with (magenta lines) and without (black lines) taking into account polarization effects. The inset plots the conduction band diagrams for different Aluminum compositions.

Figure 2: Electron density $n(z)$ in $A l_{x} G a_{1-x} N / G a N$ structures calculated at $300 K$. The aluminum composition is varying from 0.1 to 0.5 .

Figure 3: Comparative transmission coefficient profile for double barriers single well $A l_{x} G a_{1-x} N / G a N$ RTD under zero applied electric field calculated for different aluminum composition.

Figure 4: The $J-V$ curves of the double barrier $A l_{x} G a_{1-x} N / G a N$ structure simulated for different Al composition: (a) without SC effects and (b) including SC effects.

Figure 5: Conduction band profile for the $A l_{0.5} G a_{0.5} \mathrm{~N} / \mathrm{GaN}$ double barrier structure calculated for various applied bias. Solid line: SC method and dashed line: non SC method.

Figure 6: Current density versus external applied bias calculated self-consistently for different thicknesses: (a) of $A l G a N$ barrier layer and (b) of $G a N$ quantum well. 